\title{
Using quadrature and an iterative eigensolver to compute fine-structure ro-vibrational levels of Van der Waals complexes: $\mathrm{NH}\left({ }^{3} \Sigma^{-}\right)-\mathrm{He}, \mathrm{O}_{2}\left({ }^{3} \Sigma_{g}^{-}\right)-\mathrm{Ar}$ and $\mathrm{O}_{2}\left({ }^{3} \Sigma_{g}^{-}\right)-\mathrm{He}$
}

\author{
Xiao-Gang Wang* and Tucker Carrington Jr. ${ }^{\dagger}$ \\ Chemistry Department, Queen's University, \\ Kingston, Ontario KYL 3N6, Canada
}

(Dated: Apr 19, 2018; Dec 18, 2018)

\begin{abstract}
We introduce a new method for computing spectra of molecules for which a spin-spin term in the Hamiltonian has an important effect. In previous calculations, matrix elements of the spin-spin term and of the potential were obtained by expanding the potential and using analytic equations in terms of $3-j$ symbols. Instead, we use quadrature. Quadrature is simple and makes it possible to do calculations with a general potential and without using the Wigner-Eckart theorem. In previous calculations, the Hamiltonian matrix was built and diagonalized. Instead, we use an iterative eigensolver. It makes it easy to work with a large basis. The ideas are tested by computing energy levels of $\mathrm{NH}\left({ }^{3} \Sigma^{-}\right)-\mathrm{He}, \mathrm{O}_{2}\left({ }^{3} \Sigma_{g}^{-}\right)$-Ar and $\mathrm{O}_{2}\left({ }^{3} \Sigma_{g}^{-}\right)-\mathrm{He}$.
\end{abstract}

*Electronic address: xgwang.dalian@gmail.com

$\dagger$ Electronic address: Tucker. Carrington@queensu.ca 


\section{INTRODUCTION}

Multi-dimensional quadrature makes it possible to compute the ro-vibrational spectrum of a molecule, even when the potential energy surface (PES) does not have a special form that facilitates calculating integrals. Individual matrix elements could themselves be computed by using multi-dimensional quadrature, however, by using an iterative eigensolver in conjunction with multi-dimensional quadrature, spectra can be computed without calculating potential matrix elements. [1-9] It is only necessary to evaluate matrix-vector products (MVPs). A MVP transforms an input vector into an output vector. The output vector can be obtained by doing sums sequentially. This sequential summation procedure has important advantages: 1) it can be used regardless of the functional form of the PES; 2) the cost of calculating MVPs scales as it would if the PES were a sum of products (SOP), i.e., it is not more expensive to use a general PES than a SOP PES (and is less expensive unless the SOP has very few terms ); 3) there is no need to derive and use closed-form equations for complicated integrals. MVPs are evaluated efficiently by exploiting product structure imposed by using a basis of products of 1-D functions and a product quadrature grid.

In this paper, we show that a quadrature method for computing the ro-vibrational spectrum of a molecule for which interaction between rotational angular momenta and electronic spin angular momentum is important has the same advantages. We are not the first to include this interaction in a variational calculation. [10-12] Several effective fitting Hamiltonians that include such interactions have been derived and used. [13-17] In previous variational calculations, elements of a Hamiltonian matrix were computed using closed-form expressions and a Hamiltonian matrix was stored and diagonalized with a direct (not iterative) algorithm, whose cost scales as $N^{3}$, where $N$ is the size of the matrix. To do this one relies on a special representation of the PES in terms of Legendre polynomials and uses formulae involving $3-j$ symbols and reduced matrix elements to calculate matrix elements of both the PES and the spin interaction term. The method we present works with general PESs. We obviate the need for 3- $j$ symbols and concerns about phase conventions etc. by computing matrix elements of the spin interaction term and the PES with quadrature. All MVPs are done by evaluating sums sequentially. In this paper, we propose equations for a complex with three atoms $\mathrm{AB}-\mathrm{R} ; \mathrm{AB}$ is a diatomic molecule in a ${ }^{3} \Sigma$ electronic state with 
electron spin $S=1$ and $\mathrm{R}$ is a closed-shell atom with zero spin. Everything is done using a body-fixed basis that is advantageous when the anisotropy of the PES is strong. We test our equations by computing energy levels for $\mathrm{O}_{2}-\mathrm{Ar}, \mathrm{O}_{2}-\mathrm{He}$ and $\mathrm{NH}-\mathrm{He}$. Tennyson and co-workers were the first to do calculations on $\mathrm{O}_{2}$-Ar, $\mathrm{O}_{2}$-He. [10, 11] Van der Avoird and Brocks[18] have done calculations for $\mathrm{O}_{2}$ dimer.

\section{VIBRATION-ROTATION-SPIN KINETIC ENERGY OPERATOR}

To derive the correct kinetic energy operator (KEO), it is best to begin with the dimerfixed (DF) frame KEO for a complex composed of two monomers which we denote A and B. The DF frame is a two-angle body-fixed (BF) frame. It is obtained by rotating a space-fixed (SF) frame by two Euler angles $(\alpha, \beta)$ so that the DF frame z-axis is aligned with $\boldsymbol{r}_{\mathbf{0}}$, the vector connecting the centers of mass of A and B. See Fig. 1a. As shown by Brocks et al.[19], the DF KEO is

$$
\begin{aligned}
T^{\mathrm{DF}} & =T^{A}+T^{B}+T_{i n t} \\
T_{\text {int }} & =-\frac{1}{2 \mu_{0} r_{0}^{2}} \frac{\partial}{\partial r_{0}} r_{0}^{2} \frac{\partial}{\partial r_{0}}+B_{0}\left(r_{0}\right)\left[J^{2}-\cot \beta \frac{\partial}{\partial \beta}+\boldsymbol{j}^{2}-2 \boldsymbol{j} \cdot \boldsymbol{J}\right],
\end{aligned}
$$

where $B_{0}(0)=1 /\left(2 \mu_{r_{0}} r_{0}^{2}\right)$. $\mu_{0}$ is the reduced mass. $\boldsymbol{J}$ is the total angular momentum. $\boldsymbol{j}$ is the sum of the angular momenta of the two monomers. $T^{A}$ and $T^{B}$ are KEOs for monomers $\mathrm{A}$ and $\mathrm{B}$ in the DF frame. Components of $\boldsymbol{J}$ and $\boldsymbol{j}$ are in the DF frame. The components of $\boldsymbol{J}$ have a special form and are written in terms of $\alpha, \beta$ and $j_{z}$ (See Eq. (20) of Brocks et al. $[19])$.

In this paper, we consider systems in which monomer $\mathrm{A}$ is a diatomic molecule in $\mathrm{a}^{3} \Sigma_{g}^{-}$ electronic state with electron spin $S=1$ and monomer $\mathrm{B}$ is an atom. The total angular momentum of monomer $\mathrm{A}$ is

$$
j=N_{1}+S
$$

where $\boldsymbol{N}_{\mathbf{1}}$ is the rotational angular momentum associated with $\boldsymbol{r}_{\mathbf{1}}$, the diatomic vector. See Fig. 1a. $\boldsymbol{N}_{\mathbf{1}}$ is usually called $\boldsymbol{l}_{\mathbf{1}}$, if there is no spin. In the DF frame, the spherical polar angles for vector $\boldsymbol{r}_{\mathbf{1}}$ are $\left(\theta_{1}, \gamma\right)$ ( $\gamma$ is used for the azimuthal angle to be consistent with the standard three-angle BF frame whose orientation in the SF frame is specified by three Euler 
angles $(\alpha, \beta, \gamma))$. The expressions for $\boldsymbol{N}_{\mathbf{1}}$ operators in terms of $\left(\theta_{1}, \gamma\right)$ are standard, see e.g. Eq. (1.36) of Ref. 20. The spin-rovibrational KEO for monomer A is[21]

$$
T^{A}=-\frac{1}{2 \mu_{1} r_{1}^{2}} \frac{\partial}{\partial r_{1}} r_{1}^{2} \frac{\partial}{\partial r_{1}}+B_{1}\left(r_{1}\right) \boldsymbol{N}_{1}^{2}+T_{\text {fine }} .
$$

It is the usual diatomic KEO plus effective spin-spin and spin-rotation interaction terms[21],

$$
T_{\text {fine }}=\frac{2}{3} \lambda_{0}\left(3 S_{\zeta}^{2}-S^{2}\right)+\gamma_{0} \boldsymbol{N}_{\mathbf{1}} \cdot \boldsymbol{S}
$$

where $S_{\zeta}$ is the projection of $\boldsymbol{S}$ along the diatomic vector. $T^{B}=0$ because B is an atom. Substituting Eq. (2) and Eq. (3) into Eq. (1), we obtain

$$
T^{\mathrm{DF}}=T_{\mathrm{str}}+B_{0}\left(r_{0}\right)\left[J^{2}-\cot \beta \frac{\partial}{\partial \beta}+\left(\boldsymbol{N}_{\mathbf{1}}+\boldsymbol{S}\right)^{2}-2\left(\boldsymbol{N}_{\mathbf{1}}+\boldsymbol{S}\right) \cdot \boldsymbol{J}\right]+B_{1}\left(r_{1}\right) \boldsymbol{N}_{\mathbf{1}}^{2}+T_{\text {fine }},
$$

where

$$
T_{\text {str }}=-\frac{1}{2 \mu_{0} r_{0}^{2}} \frac{\partial}{\partial r_{0}} r_{0}^{2} \frac{\partial}{\partial r_{0}}-\frac{1}{2 \mu_{1} r_{1}^{2}} \frac{\partial}{\partial r_{1}} r_{1}^{2} \frac{\partial}{\partial r_{1}} .
$$

In this paper, the diatomic is rigid and therefore the second term in Eq. (6) is removed. In summary, to derive the KEO that includes coupling between spin and rotation, one replaces $\boldsymbol{j}$ in $T_{i n t}$ with $\boldsymbol{N}_{\mathbf{1}}+\boldsymbol{S}$, but uses $\boldsymbol{N}_{\mathbf{1}}$ and not $\boldsymbol{N}_{\mathbf{1}}+\boldsymbol{S}$ in $T^{A}$. $T_{\text {fine }}$ also couples spin and rotation.

\section{BASIS AND MATRIX ELEMENTS}

Knowing the KEO and having a potential, we calculate vibration-rotation-spin energy levels by choosing a basis and evaluating matrix-vector products (MVPs) to use the Lanczos

algorithm. When spin effects are omitted this is a common procedure. [2, 6, 22-24] When the Lanczos vectors are not orthogonalized, the memory and CPU costs are low. To calculate wavefunctions we repeat the Lanczos iteration. [25-27] The vibration-rotation-spin basis is a direct product of a stretch basis and a bend-rotation-spin basis. The stretch basis functions are discrete variable representation (DVR) functions. [2] In the rest of the section, we discuss only the bend-rotation-spin basis. Each bend-rotation-spin basis function is a product,

$$
\left|N_{1} m_{N_{1}} ; J K M ; S m_{S}\right\rangle=Y_{N_{1}}^{m_{N_{1}}}\left(\theta_{1}, \gamma\right) \sqrt{\frac{2 J+1}{4 \pi}} D_{M K}^{J}(\alpha, \beta, 0)^{*}\left|S m_{S}\right\rangle
$$


with $K \equiv m_{N_{1}}+m_{S} \cdot m_{N_{1}}, m_{S}$, and $K$ are projection quantum numbers, along the dimerfixed (DF) z-axis, of the rotational angular momentum $\boldsymbol{N}_{\mathbf{1}}$, the electronic spin $\boldsymbol{S}$, and the total angular momentum $\boldsymbol{J}$. Note that for a triatomic molecule with no spin, we normally use $\left|l_{1} m_{1}\right\rangle$ instead of $\left|N_{1} m_{N_{1}}\right\rangle$ for the angular basis associated with $\boldsymbol{r}_{\mathbf{1}}$. In this paper, we choose to use $\left|N_{1} m_{N_{1}}\right\rangle$ because we include the electronic spin angular momentum for the diatomic. The basis of Eq. (7) can be written in terms of a standard symmetric top Wigner function,

$$
\left|N_{1} m_{N_{1}} ; J K M ; S m_{S}\right\rangle=\Theta_{N_{1}}^{m_{N_{1}}}\left(\theta_{1}\right) \sqrt{\frac{2 J+1}{8 \pi^{2}}} D_{M K}^{J}(\alpha, \beta, \gamma)^{*} e^{-i m_{S} \gamma}\left|S m_{S}\right\rangle
$$

because $K \equiv m_{N_{1}}+m_{S}$. If $S=0$, Eq. (8) is the standard triatomic vibration-rotation basis. [28] $Y_{N_{1}}^{m_{N_{1}}}\left(\theta_{1}, \gamma\right)=\Theta_{N_{1}}^{m_{N_{1}}}\left(\theta_{1}\right)(1 / \sqrt{2 \pi}) e^{i m_{N_{1}} \gamma}$ is a spherical harmonic function with $\Theta_{N_{1}}^{m_{N_{1}}}\left(\theta_{1}\right)$ being an associated Legendre function. Dubernet et al. [29]. define angular momentum coupling cases, analogous to Hund's case for diatomic molecules, for the diatomics of Van der Waals complexes composed of an open-shell diatomic and an atom. The basis of Eq. (7) corresponds to their case A, which is analogous to Hund's case a. To understand why case A of Dubernet et al. corresponds to Hund's case a, consider starting with Eq. (7) and making monomer $\mathrm{B}$ an atom. This means removing $\theta_{1}$ and the labels $N_{1}$ and $m_{N_{1}}$, and replacing the constraint with $K \equiv m_{S}$. Eq. (7) then becomes the Hund's case (a) basis. [30, 31].

Tennyson and Mettes[10] did the first variational calculation for $\mathrm{O}_{2}$-Ar. They used the SF frame basis, $\left|((N S) j L) J M_{J}\right\rangle$, in the notation of Ref. 10, with $M_{J}$ the SF Z-axis projection of total $J$. Their basis was built by successively coupling pairs of angular momenta with Clebsch-Gordan coefficients. In our notation, this basis would be $\left|\left(\left(N_{1} S\right) j l_{0}\right) J M\right\rangle$. A notable difference between their basis and our BF basis, Eq. (7), is that the BF basis does not have the $l_{0}$ quantum number because the $z$-axis of the BF frame is along $\boldsymbol{r}_{\mathbf{0}}$. The BF basis also does not have the $j$ label. It does have projection quantum numbers along the BF z-axis. To analytically evaluate potential integrals in their SF basis, Tennyson and Mettes expand the potential in terms of Legendre polynomials. In the SF basis, equations for the matrix elements of $T_{\text {fine }}$ are known in terms of $3-j$ and $6-j$ symbols[21]. They are derived with the Wigner-Eckart theorem.

We are not the first to propose using the BF basis of Eq. (7). Van der Avoird used it in Ref. 12, with perturbation theory, to explain the variational results of Tennyson and 
Mettes[10]. Using the BF projection quantum numbers $K$ and $m_{N_{1}}$, Van der Avoird identified ladder patterns in the variational levels. Using the Wigner-Eckart theorem[21], he derived analytic expressions for matrix elements of $T_{\text {fine }}$ in the BF basis. These expressions involve a $3-j$ symbol and double-bar reduced matrix elements. In section IV, we will propose a new method for calculating matrix elements of $T_{\text {fine }}$ in the BF basis. Van der Avoird and Brocks[18] use a $\mathrm{BF}$ basis to variationally compute fine-structure levels of $\mathrm{O}_{2}$ dimer, a problem more challenging than the triatomic in this paper.

As mentioned above, operators for the components of $\boldsymbol{J}$ in the DF frame have a special form. Nonetheless, if $m_{N_{1}}$ in Eq. (7) is equal to $K-m_{S}$, one obtains

$$
\begin{aligned}
\hat{j}_{z}\left|N_{1} m_{N_{1}} ; J K M ; S m_{S}\right\rangle & =\left(\hat{N}_{1 z}+\hat{S}_{z}\right)\left|N_{1} m_{N_{1}} ; J K M ; S m_{S}\right\rangle \\
& =\left(m_{N_{1}}+m_{S}\right)\left|N_{1} m_{N_{1}} ; J K M ; S m_{S}\right\rangle \\
& =K\left|N_{1} m_{N_{1}} ; J K M ; S m_{S}\right\rangle .
\end{aligned}
$$

Because of this equation, the matrix elements of $\boldsymbol{J}$ operators in the DF frame can be obtained from the usual standard equations, as in the case of systems with no spin. [32] $J^{2}-\cot \beta \partial / \partial \beta$ is diagonal and its non-zero elements are $J(J+1)$. Matrix elements of $J_{ \pm}$are also equal to the usual expressions. [20] The constraint $K=m_{N_{1}}+m_{S}$ has a physical interpretation. As we see from Fig. 1a, the constraint arises because $\boldsymbol{J}=\boldsymbol{l}_{\mathbf{0}}+\boldsymbol{N}_{\mathbf{1}}+\boldsymbol{S}$ and $\boldsymbol{l}_{0}$ has no projection on the z-axis of the DF frame.

Non-zero diagonal KEO matrix elements, in the basis of Eq. (7), for all terms except the $S_{\zeta}^{2}$ term, which we shall focus on later in the paper, are:

$$
\begin{aligned}
& \left\langle N_{1} m_{N_{1}} ; J K M ; S m_{S}\left|T^{\mathrm{DF}}\right| N_{1} m_{N_{1}} ; J K M ; S m_{S}\right\rangle \\
= & B_{0}\left[J(J+1)+N_{1}\left(N_{1}+1\right)+S(S+1)+2 m_{N_{1}} m_{S}-2 K^{2}\right] \\
& -\frac{2}{3} \lambda_{0} S(S+1)+\gamma_{0} m_{N_{1}} m_{S},
\end{aligned}
$$

where the last two terms are from $T_{\text {fine }}$ KEO terms that are off-diagonal are $\boldsymbol{N}_{\mathbf{1}} \cdot \boldsymbol{J}, \boldsymbol{S} \cdot \boldsymbol{J}$, and $\boldsymbol{N}_{\mathbf{1}} \cdot \boldsymbol{S}$ from $T_{\text {int }}$ and $\boldsymbol{N}_{\mathbf{1}} \cdot \boldsymbol{S}$ from $T_{\text {fine. }}$. Their matrix elements are computed from the 
relations,

$$
\begin{aligned}
\boldsymbol{N}_{\mathbf{1}} \cdot \boldsymbol{J} & =\frac{1}{2}\left(N_{1+} J_{-}+N_{1-} J_{+}+2 N_{1 z} J_{z}\right) \\
\boldsymbol{S} \cdot \boldsymbol{J} & =\frac{1}{2}\left(S_{+} J_{-}+S_{-} J_{+}+2 S_{z} J_{z}\right) \\
\boldsymbol{N}_{\mathbf{1}} \cdot \boldsymbol{S} & =\frac{1}{2}\left(N_{1+} S_{-}+N_{1-} S_{+}+2 N_{1 z} S_{z}\right),
\end{aligned}
$$

where $N_{1 \pm}=N_{1 x} \pm i N_{1 y}, J_{ \pm}=J_{x} \pm i J_{y}$, and $S_{ \pm}=S_{x} \pm i S_{y}$. Excluding the $S_{\zeta}^{2}$ term, the non-zero off-diagonal matrix elements are

$$
\begin{aligned}
N_{1+} J_{-}: & \left\langle N_{1}, m_{N_{1}}+1 ; J, K+1, M ; S m_{S}\left|T^{\mathrm{DF}}\right| N_{1} m_{N_{1}} ; J K M ; S m_{S}\right\rangle=-B_{0} \lambda_{N_{1}, m_{N_{1}}}^{+} \lambda_{J K}^{+} \\
S_{+} J_{-}: & \left\langle N_{1}, m_{N_{1}} ; J, K+1, M ; S m_{S}+1\left|T^{\mathrm{DF}}\right| N_{1} m_{N_{1}} ; J K M ; S m_{S}\right\rangle=\left(-B_{0}\right) \lambda_{S, m_{S}}^{+} \lambda_{J K}^{+} \\
N_{1-} S_{+}: & \left\langle N_{1}, m_{N_{1}}-1 ; J, K, M ; S m_{S}+1\left|T^{\mathrm{DF}}\right| N_{1} m_{N_{1}} ; J K M ; S m_{S}\right\rangle=\left(B_{0}+\frac{1}{2} \gamma_{0}\right) \lambda_{N_{1}, m_{N_{1}}}^{-} \lambda_{S m_{S}}^{+}
\end{aligned}
$$

the operator responsible for the off-diagonal element is given at the beginning of each line and $\lambda_{J K}^{ \pm}=\sqrt{J(J+1)-K(K \pm 1)}$ etc.

\section{A. Parity-adapted basis}

We prefer to work with a parity-adapted uncoupled basis which has the advantage of being two times smaller. The parity-adapted basis functions are equal to or linear combinations of the uncoupled basis functions,

$$
\begin{aligned}
& u_{N_{1} m_{N_{1}} K m_{S}}^{J M S P}=N_{K m_{S}} \frac{1}{\sqrt{2}} \times \\
& {\left[\left|N_{1} m_{N_{1}} ; J K M\right\rangle\left|S m_{S}\right\rangle+(-1)^{J+S+P}\left|N_{1} \bar{m}_{N_{1}} ; J \bar{K} M\right\rangle\left|S \bar{m}_{S}\right\rangle\right],}
\end{aligned}
$$

with

$$
N_{K m_{S}}=\left(1+\delta_{K, 0} \delta_{m_{S}, 0}\right)^{-1 / 2}
$$

where $P=0$ and 1 correspond to even and odd parity, respectively. The parity adapted basis functions are obtained by considering,

$$
E^{*}\left|N_{1} m_{N_{1}} ; J K M\right\rangle\left|S m_{S}\right\rangle=(-1)^{J+S}\left|N_{1} \bar{m}_{N_{1}} ; J \bar{K} M\right\rangle\left|S \bar{m}_{S}\right\rangle
$$


which is obtained from $E^{*}\left|S m_{S}\right\rangle=(-1)^{S}\left|S \bar{m}_{S}\right\rangle$ (See Eq. (A18) of Ref. 33) and $E^{*}\left|N_{1} m_{N_{1}} ; J K M\right\rangle=(-1)^{J}\left|N_{1} \bar{m}_{N_{1}} ; J \bar{K} M\right\rangle$ (See e.g. Eq. 10 of Ref. 34). Note that the ${ }^{3} \Sigma^{-}$electronic wavefunction has odd parity. This factor is not included in Eq. (14) and not included in our tables and figures. In this work, we label levels with their spectroscopic parity $e / f$, which is $(-1)^{J+P}= \pm 1$, respectively. For a single parity, the number of parityadapted functions is roughly half the number of non-parity-adapted basis functions. The parity-adapted and non-parity-adapted bases span the same space if the quantum numbers are restricted by

$$
\begin{aligned}
m_{S} & \geq 0, \\
K & \geq 0 \quad\left(\text { if } m_{S}=0\right) .
\end{aligned}
$$

These restrictions complicate the evaluation of MVPs in the parity-adapted basis and we therefore transform from the PA basis to the basis of Eq. (7), evaluate the MVP in that basis, and then transform back to the PA basis. Recall that $m_{N_{1}}$ is determined by $m_{S}$ and $K$ and is not an independent basis label.

Van der Avoird also used a parity-adapted basis in Eq. 12 of Ref. 12. His notation $(-1)^{p}$ is equivalent to our $(-1)^{J+S+P}$ in Eq. (13). This is confirmed by comparing the assignments of our $\mathrm{O}_{2}$-Ar levels, reported in section VIII, with those of Ref. 12.

\section{MATRIX ELEMENTS OF THE SPIN INTERACTION TERM $S_{\zeta}^{2}$}

We use the basis of Eq. (7) because when the anisotropy of the PES is strong it is an excellent basis. Unfortunately, it is not simple to apply the spin interaction term to one of the basis functions. The problem is that the spin interaction term $T_{\text {fine }}$ is written in terms

of $S_{\zeta}$, the component of the spin operator along the diatomic axis, and the basis label $m_{S}$ is the quantum number for the $z$ component of the spin operator along the DF $z$ axis which is inter-monomer Jacobi vector. In order to calculate matrix elements of the spin interaction term, we write each of the basis functions in terms of functions of a basis in which $S_{\zeta}^{2}$ is diagonal. The relation between these two spin bases is[20]

$$
\left|S m_{S}\right\rangle=\sum_{\Sigma=-1}^{+1} D_{m_{S} \Sigma}^{1 *}\left(\gamma, \theta_{1}, 0\right)|S \Sigma\rangle=e^{i m_{S} \gamma} \sum_{\Sigma=-1}^{+1} d_{m_{S} \Sigma}^{1}\left(\theta_{1}\right)|S \Sigma\rangle
$$


where $\Sigma$ is the component along the diatomic axis and $d$ is Wigner small d-function[20]. Now, a $S_{\zeta}^{2}$ matrix element can be evaluated by doing a sum,

$$
\begin{aligned}
& \left\langle N_{1}^{\prime} m_{N_{1}}^{\prime} ; J K^{\prime} M ; S m_{S}^{\prime}\left|S_{\zeta}^{2}\right| N_{1} m_{N_{1}} ; J K M ; S m_{S}\right\rangle \\
& =\sum_{\Sigma=-1}^{+1} \Sigma^{2}\left\langle N_{1}^{\prime} m_{N_{1}}^{\prime} ; J K^{\prime} M ; S m_{S}^{\prime}\left|e^{-i m_{S}^{\prime} \gamma} e^{i m_{S} \gamma} d_{m_{S}^{\prime} \Sigma}^{1}\left(\theta_{1}\right) d_{m_{S} \Sigma}^{1}\left(\theta_{1}\right)\right| N_{1} m_{N_{1}} ; J K M ; S m_{S}\right\rangle .
\end{aligned}
$$

The integral in Eq. (17) is diagonal in $M$ because the operator in the middle does not depend on the Euler angle $\alpha$. We now show that the integral in Eq. (17) is also diagonal in $K$. In Eq. (17), the integral over $\gamma$ is

$$
\int_{0}^{2 \pi} d \gamma \frac{1}{\sqrt{2 \pi}} e^{-i\left(m_{N_{1}}^{\prime}+m_{S}^{\prime}\right) \gamma} \frac{1}{\sqrt{2 \pi}} e^{i\left(m_{N_{1}}+m_{S}\right) \gamma}=\delta_{K^{\prime}, K}
$$

because $m_{N_{1}}+m_{S}=K$. Without the factor $e^{i m_{S} \gamma}$ in Eq. (16), Eq. (17) would not be diagonal in $K$. Owing to the diagonality in $K$, the integrand of the integral over $\beta$ in Eq. (17) is a product of two orthonormal functions, $d_{M, K}^{J}(\beta) d_{M, K}^{J}(\beta)$ and is therefore unity. We can therefore re-write Eq. (17) as,

$$
\begin{aligned}
& \left\langle N_{1}^{\prime} m_{N_{1}}^{\prime} ; J K M ; S m_{S}^{\prime}\left|S_{\zeta}^{2}\right| N_{1} m_{N_{1}} ; J K M ; S m_{S}\right\rangle \\
& =\sum_{\Sigma=-1}^{+1} \Sigma^{2} \int_{0}^{\pi} d \theta_{1} \sin \theta_{1} \Theta_{N_{1}^{\prime}}^{m_{N_{1}}^{\prime}}\left(\theta_{1}\right) d_{m_{S}^{\prime} \Sigma}^{1}\left(\theta_{1}\right) d_{m_{S} \Sigma}^{1}\left(\theta_{1}\right) \Theta_{N_{1}}^{m_{N_{1}}}\left(\theta_{1}\right) \\
& =\sum_{\Sigma=-1}^{+1} \Sigma^{2} \sum_{\alpha_{1}} T_{N_{1}^{\prime}, \alpha_{1}}^{m_{N_{1}}^{\prime}} d_{m_{S}^{\prime} \Sigma}^{1}\left(\theta_{\alpha_{1}}\right) d_{m_{S} \Sigma}^{1}\left(\theta_{\alpha_{1}}\right) T_{N_{1}, \alpha_{1}}^{m_{N_{1}}} \\
& =\sum_{\alpha_{1}} T_{N_{1}^{\prime}, \alpha_{1}}^{m_{N_{1}}^{\prime}} A_{m_{S}^{\prime} m_{S}, \alpha_{1}} T_{N_{1}, \alpha_{1}}^{m_{N_{1}}},
\end{aligned}
$$

with

$$
A_{m_{S}^{\prime} m_{S}, \alpha_{1}}=\sum_{\Sigma=-1}^{+1} \Sigma^{2} d_{m_{S}^{\prime} \Sigma}^{1}\left(\theta_{\alpha_{1}}\right) d_{m_{S} \Sigma}^{1}\left(\theta_{\alpha_{1}}\right)
$$

and

$$
T_{N_{1}, \alpha_{1}}^{m_{N_{1}}}=\sqrt{w_{\alpha_{1}}} \Theta_{N_{1}}^{m_{N_{1}}}\left(\theta_{\alpha_{1}}\right)
$$

where $\left(w_{\alpha_{1}}, \theta_{\alpha_{1}}\right)$ are Gauss-Legendre quadrature weights and points. $N_{1}, m_{N_{1}}$ and $m_{S}$ are coupled by the spin term. The sum over $\alpha_{1}$ in Eq. (19) has almost the same form as a standard quadrature approximation for a matrix element of a 1-D $\theta$ potential in an associated Legendre basis. However in Eq. (19), $A_{m_{S}^{\prime} m_{S}, \alpha_{1}}$ is off-diagonal in $m_{S}$. For a particular $\left(m_{S}^{\prime}, m_{S}\right)$ pair, 
$A_{m_{S}^{\prime} m_{S}, \alpha_{1}}$ is a sum of terms $\sin ^{n_{s}} \theta \cos ^{n_{c}} \theta$, where $n_{c}+n_{s} \leq 2$. Despite the fact that there are terms with $n_{s}=1$, we use Gauss Legendre quadrature. It is nearly exact when the number of points is chosen to be large enough to converge potential integrals. A matrix-vector product with the $S_{\zeta}^{2}$ matrix in Eq. (19) can be evaluated by doing sums sequentially,

$$
x_{N_{1}^{\prime} m_{N_{1}}^{\prime}, J K, S m_{S}^{\prime}}^{\prime}=\sum_{\alpha_{1}} T_{N_{1}^{\prime}, \alpha_{1}}^{m_{N_{1}}^{\prime}} \sum_{m_{S}} A_{m_{S}^{\prime} m_{S}, \alpha_{1}} \sum_{N_{1}} T_{N_{1}, \alpha_{1}}^{m_{N_{1}}} x_{N_{1} m_{N_{1}}, J K, S m_{S}},
$$

where $x_{N_{1} m_{N_{1}}, J K, S m_{S}}$ is a vector in the basis of Eq. (7). Because of the constraint, $m_{N_{1}}$ on the input vector is not an independent label and is computed from $m_{N_{1}}=K-m_{S}$, and $m_{N_{1}}^{\prime}$ on the output vector is not an independent label and is computed from $m_{N_{1}}^{\prime}=K-m_{S}^{\prime}$.

Rather than using Eq. (22), one could calculate the matrix elements on the left side of Eq. (19), by doing the sum over $\alpha_{1}$. After doing the sum over $\alpha_{1}$, one would obtain the same numbers that Van der Avoird gets using his analytic equation in terms of $3-j$ symbols and double bar reduced matrix elements. His equation shows that $N_{1}$ is coupled only to $N_{1}^{\prime}=N+\Delta N_{1}$ with $\Delta N_{1} \leq 2$. The memory cost of storing the non-zero elements of the $S_{\zeta}^{2}$ matrix therefore scales as $N_{\text {bas }}$, where $N_{\text {bas }}$ is the size of the basis. One could use stored non-zero elements of the $S_{\zeta}^{2}$ matrix to compute the matrix-vector product required to use an iterative eigensolver. The cost of the MVP would then scale as $N_{\text {bas. }}$. We prefer to store the small A matrix of Eq. (20) and use sequential sums, as in Eq. (22), to compute the matrix-vector product For our choice, the memory cost is smaller and the cost of the matrix-vector product is similar. Not using analytic equations for matrix elements when it is possible might seem less elegant, but quadrature reduces the memory cost and (not in this case but in general) reduces the cost of evaluating MVPs. This is strikingly obvious for potential MVPs. Quadrature is not approximate and does not increase the cost of the calculations Moreover, the quadrature approach would also be straightforward even if $T_{\text {fine }}$ involved complicated functions of $S_{\zeta}$ operators and even if the angle between the $\zeta$ axis and the $\mathrm{BF} z$ axis were a complicated function of the shape of the molecule. It also has the advantage that one does not need to be careful about phase factor errors in analytical expressions. 


\section{ASSIGNMENT OF QUANTUM NUMBERS}

$J$ and $P$ are conserved quantum numbers and we calculate levels with different values separately. It is fairly straightforward to assign values of $K, m_{S}, m_{N_{1}}$, and $N_{1}$ to every energy level because the basis functions have these labels. To assign values of $l_{0}$ and $j_{0}$ is harder because the basis functions do not have these labels. The assignments of projection quantum numbers $K, m_{S}, m_{N_{1}}$ are made with the approach of Ref. 9. For example, $K$ is assigned by summing the squares of expansion coefficients in the non-PA basis over all indices other than $K$ to obtain a quantity we call $P_{K}$, which is a measure of the contribution of basis functions labelled by $K$ to the full wavefunction. $\left(\sum_{K} P_{K}=1\right.$.) $P_{K}$ and $P_{-K}$ are equal and $P_{m_{N_{1}}}$ and $P_{-m_{N_{1}}}$ are equal. We therefore cannot assign the signs of $K, m_{S}$, and $m_{N_{1}}$, however, using the relation, $K=m_{N_{1}}+m_{S}$, we can determine whether $m_{S}$, and $m_{N_{1}}$ have the same or different signs. For $\mathrm{O}_{2}-\mathrm{Ar}$ or $\mathrm{O}_{2}-\mathrm{He}$, this allows us to determine whether the $\mathrm{DF} z$ projections for the rotation of $\mathrm{O}_{2}$ and the spin of $\mathrm{O}_{2}$ are aligned or anti-aligned.

We also assign $l_{0}, j$ and $N_{1}$ by computing expectation values of $\boldsymbol{l}_{\mathbf{0}}^{\mathbf{2}}, \boldsymbol{j}^{\mathbf{2}}$ and $\boldsymbol{N}_{\mathbf{1}}^{\mathbf{2}}$, denoted by $\left\langle\boldsymbol{l}_{\mathbf{0}}^{\mathbf{2}}\right\rangle,\left\langle\boldsymbol{j}_{\mathbf{0}}^{\mathbf{2}}\right\rangle$, and $\left\langle\boldsymbol{N}_{\mathbf{1}}^{\mathbf{2}}\right\rangle$, respectively, for every state. These are useful approximate good quantum numbers if coupling is weak. For example, the expectation value of $\boldsymbol{N}_{\mathbf{1}}^{\mathbf{2}}$ in state $\psi^{i}$ is

$$
\begin{aligned}
\left\langle\boldsymbol{N}_{\mathbf{1}}^{\mathbf{2}}\right\rangle & =\left\langle\psi^{i}\left|N_{1}^{2}\right| \psi^{i}\right\rangle \\
& =\sum_{\substack{N_{1}^{\prime}, K^{\prime}, m_{S}^{\prime} \\
N_{1}, K, m_{S}}} C_{N_{1}^{\prime}, K^{\prime}, m_{S}^{\prime}}^{i} C_{N_{1}, K, m_{S}}^{i}\left\langle N_{1}^{\prime} m_{N_{1}}^{\prime} ; J K M ; S m_{S}^{\prime}\left|N_{1}^{2}\right| N_{1} m_{N_{1}} ; J K M ; S m_{S}\right\rangle \\
& =\sum_{N_{1}, K, m_{S}}\left(C_{N_{1}, K, m_{S}}^{i}\right)^{2} N_{1}\left(N_{1}+1\right),
\end{aligned}
$$

where

$$
\left|\psi^{i}\right\rangle=\sum_{N_{1}, K, m_{s}} C_{N_{1}, K, m_{s}}^{i}\left|N_{1} m_{N_{1}} ; J K M ; S m_{S}\right\rangle
$$

The coefficients, $C_{N_{1}, K, m_{s}}^{i}$ in the non-PA basis can be easily obtained from the coefficients in the PA basis used to do the calculation. We use the non-PA basis because its off-diagonal elements are easier to deal with (in the PA basis they are complicated due to the restrictions on the quantum numbers in Eq. (15)). Computing $\left\langle\boldsymbol{N}_{\mathbf{1}}^{\mathbf{2}}\right\rangle$ is easy because the basis functions are labelled by $N_{1}$. Computing $\left\langle\boldsymbol{l}_{\mathbf{0}}^{\mathbf{2}}\right\rangle$ and $\left\langle\boldsymbol{j}^{\mathbf{2}}\right\rangle$ is not as straightforward because our basis 
functions do not have $l_{0}$ and $j_{0}$ labels. A good way to calculate $\left\langle\boldsymbol{l}_{\mathbf{0}}^{\mathbf{2}}\right\rangle$ is to use $\boldsymbol{l}_{\mathbf{0}}^{\mathbf{2}}=\left(\boldsymbol{J}-\boldsymbol{N}_{\mathbf{1}}-\right.$ $\boldsymbol{S}^{\mathbf{2}}$. The required matrix elements are given in Eqs. 10 and 12. For $\left\langle\boldsymbol{j}^{\mathbf{2}}\right\rangle$, we compute the expectation values of $\boldsymbol{j}^{2}=\left(\boldsymbol{N}_{1}+\boldsymbol{S}\right)^{2}$. Unlike $\boldsymbol{N}_{1}^{2}$, off-diagonal matrix elements contribute to the sums for $\boldsymbol{l}_{\mathbf{0}}^{\mathbf{2}}$ and $\boldsymbol{j}^{\mathbf{2}}$.

\section{AN ALTERNATIVE METHOD OF COMPUTING THE SPECTRUM}

In the previous sections of this paper, we propose (method I) using quadrature with a body-fixed KEO whose $z$ axis is along the inter-monomer Jacobi vector from the centre of mass of $\mathrm{O}_{2}$ to the rare gas atom, in order to treat the spin interaction term proportional to $S_{\zeta}^{2}$. In this section we outline a different method (method II) that does not require any quadrature and uses a body-fixed KEO whose $z$ axis is along the diatomic $\mathrm{O}_{2}$ vector. This alternative method has disadvantages, but can be used to check calculations. In method I, quadrature is necessary because the spin basis used to compute the spectrum, $\left|S m_{S}\right\rangle$, has the label $m_{S}$ for the projection along the inter-monomer Jacobi vector and must therefore be replaced with a linear combination of spin functions, $|S \Sigma\rangle$, labelled by $\Sigma$ (Eq. (16)), the projection along the diatomic Jacobi vector, in which $S_{\zeta}^{2}$ is diagonal. In method II, the inter-monomer Jacobi vector is $\boldsymbol{r}_{\mathbf{1}}$ and the diatomic Jacobi vector is $\boldsymbol{r}_{\mathbf{0}}$. In our notation, the BF $z$ axis is along $\boldsymbol{r}_{\mathbf{0}}$. The obvious advantage of method II is that $S_{\zeta}^{2}$ is diagonal, i.e. $S_{\zeta}^{2}\left|S m_{S}\right\rangle=m_{S}^{2}\left|S m_{S}\right\rangle$ : there is no need to introduce a second set of spin functions.

An important disadvantage of method II is that the Coriolis coupling is much larger than for method I. Take NH-He as an example, the rotational constants are: $b_{\mathrm{NH}}=16.343 \mathrm{~cm}^{-1}$ and $b_{\text {inter }}=0.475 \mathrm{~cm}^{-1}$ (both evaluated at the equilibrium geometry on the PES used in this work). In method I, $B_{0}=b_{\text {inter }}=0.475 \mathrm{~cm}^{-1}$ and in method II, $B_{0}=b_{\mathrm{NH}}=16.343 \mathrm{~cm}^{-1}$. It is clear that the Coriolis interaction term, proportional to $B_{0}$, is much larger in method II. A second and perhaps more critical disadvantage of method II is that it is difficult to apply it to larger systems such as $\mathrm{O}_{2}$ - $\mathrm{CO}$ where the second monomer is a closed-shell spinless diatomic molecule rather than an atom. This is because if the $\mathrm{BF} z$ is along the $\mathrm{O}_{2}$ vector then one needs polyspherical angles for $\mathrm{CO}$ that are angles relating the orientation of two unconnected monomers and in terms of these angles potential coupling is large.

The KEO in method II has the same form as the KEO in method I. However, $r_{0}$ is now the 
diatomic Jacobi vector and $r_{1}$ is now the inter-monomer Jacobi vector. The basis functions are formally identical to those of method I, Eq. (7). Although $\boldsymbol{r}_{\mathbf{1}}$ is now the inter-monomer Jacobi vector and using $\boldsymbol{N}_{\mathbf{1}}$ for its angular momentum is unconventional, we prefer not to change the notation. The KEO terms $T^{\mathrm{DF}}, T_{\text {int }}$ and $T^{A}$ take the same form. The only formal change in the KEO is in the spin-rotation interaction term, the $\gamma_{0}$-related term (cf. Eq. (4)),

$$
T_{\text {fine }}=\frac{2}{3} \lambda_{0}\left(3 S_{\zeta}^{2}-S^{2}\right)+\gamma_{0}\left(\boldsymbol{J}-\boldsymbol{N}_{\mathbf{1}}-\boldsymbol{S}\right) \cdot \boldsymbol{S}
$$

This change is necessary because in method I the rotation of the diatomic molecule with spin is described by $\boldsymbol{r}_{\mathbf{1}}$ and its angular momentum $\boldsymbol{N}_{\mathbf{1}}$ and in method II the rotation of the diatomic molecule with spin is described by $\boldsymbol{r}_{\mathbf{0}}$ and its angular momentum $\boldsymbol{l}_{\mathbf{0}}=\boldsymbol{J}-\boldsymbol{N}_{\mathbf{1}}-\boldsymbol{S}$. Because of this change, the diagonal and off-diagonal matrix elements change slightly. Nonzero diagonal KEO matrix elements, in the basis of Eq. (7), for all terms including the $S_{\zeta}^{2}$ term, are:

$$
\begin{aligned}
& \left\langle N_{1} m_{N_{1}} ; J K M ; S m_{S}\left|T^{\mathrm{DF}}\right| N_{1} m_{N_{1}} ; J K M ; S m_{S}\right\rangle \\
= & B_{0}\left[J(J+1)+N_{1}\left(N_{1}+1\right)+S(S+1)+2 m_{N_{1}} m_{S}-2 K^{2}\right] \\
& +2 \lambda_{0} m_{S}^{2}-\frac{2}{3} \lambda_{0} S(S+1)-\gamma_{0}\left[S(S+1)+m_{S}^{2}\right] .
\end{aligned}
$$

Compared to Eq. (10), the new term $2 \lambda_{0} m_{S}^{2}$ is from the $2 \lambda_{0} S_{\zeta}^{2}$ term of $T_{\text {fine }}$ and the $\gamma_{0}$-related term has changed. Eq. (10) does not have the $2 \lambda_{0} S_{\zeta}^{2}$ term.

Including the $S_{\zeta}^{2}$ term, the non-zero off-diagonal matrix elements are

$$
\begin{aligned}
N_{1+} J_{-}: & \left\langle N_{1}, m_{N_{1}}+1 ; J, K+1, M ; S m_{S}\left|T^{\mathrm{DF}}\right| N_{1} m_{N_{1}} ; J K M ; S m_{S}\right\rangle=-B_{0} \lambda_{N_{1}, m_{N_{1}}}^{+} \lambda_{J K}^{+} \\
S_{+} J_{-}: & \left\langle N_{1}, m_{N_{1}} ; J, K+1, M ; S m_{S}+1\left|T^{\mathrm{DF}}\right| N_{1} m_{N_{1}} ; J K M ; S m_{S}\right\rangle=-\left(B_{0}-\frac{1}{2} \gamma_{0}\right) \lambda_{S, m_{S}}^{+} \lambda_{J K}^{+} \\
N_{1-} S_{+}: & \left\langle N_{1}, m_{N_{1}}-1 ; J, K, M ; S m_{S}+1\left|T^{\mathrm{DF}}\right| N_{1} m_{N_{1}} ; J K M ; S m_{S}\right\rangle=\left(B_{0}-\frac{1}{2} \gamma_{0}\right) \lambda_{N_{1}, m_{N_{1}}}^{-} \lambda_{S m_{S}}^{+},
\end{aligned}
$$

with the corresponding operator given at the beginning of each line. Compared to Eq. (12), the second and third equations are different due to the change of the $\gamma_{0}$-related term in the KEO. 


\section{RESULTS: SPIN-ROVIBRATIONAL LEVELS OF NH( $\left.{ }^{3} \Sigma^{-}\right)$-HE}

Using both method I and method II, we computed the spin-rovibration levels of $\mathrm{NH}\left({ }^{3} \Sigma^{-}\right)$He, which were previously reported in Ref. 35. Cybulski et al. also used two methods: the SF approach of Tennyson and Mettes[10, 11] and a BF method with the same basis we have in method I (the $z$ angular momentum and spin components that label basis functions are along the inter-monomer Jacobi vector). The two sets of energies computed by Cybulski et al. differ by less than $0.0001 \mathrm{~cm}^{-1}$. Their accurate calculations allow us to test our methods. We use the PES "potential I" of Ref. 35 and the same parameters: $b_{\mathrm{NH}}=16.343 \mathrm{~cm}^{-1}$ is the rotational constant of $\mathrm{NH}$ in its ground vibrational state; $\lambda_{0}=0.920 \mathrm{~cm}^{-1}$ and $\gamma_{0}=-0.055$ $\mathrm{cm}^{-1}$ are, respectively, the spin-spin and spin-rotation interaction constants for $\mathrm{NH}$. We also use the same atomic masses, as in Ref. 35. The NH distance is fixed throughout the calculations.

The size of the bend basis is determined by $\max \left(N_{1}\right)=25$; Cybulski et al. used a maximum value of 8 . 30 Gauss-Legendre quadrature points are used for $\theta_{1}$, to integrate the potential and $S_{\zeta}^{2}$ term in Eq. 19. The stretch basis for $r_{0}$ is 120 sine DVR functions in the range $[3.5,35] a_{0}$. The total basis size is small enough that when using an iterative eigensolver, there is no need to optimise the stretch basis.

Cybulski et al. calculate levels with and without $T_{\text {fine }}$. The two sets of levels are labelled "no spin" and "with spin" in Table I. "No spin" levels with $l_{0}>0$ are split into three closely spaced "with spin" levels. For their "no spin" levels Cybulski et al. use the dissociation limit on their PES as the zero of energy. For their "with spin" levels they use a different zero of energy. The zero of energy for "with spin" is the experimental[36] energy of the lowest rotational-spin $\mathrm{NH}\left({ }^{3} \Sigma^{-}\right)$level. It is $0.0077 \mathrm{~cm}^{-1}[35,36]$ below the dissociation energy on the PES. This $0.0077 \mathrm{~cm}^{-1}$ shift is due to $T_{\text {fine }}$. Our levels are compared to those of Cybulski et

al. in Table I. For both our "no spin" and our "with spin" levels, the zero of energy is the dissociation limit. To compare Cybulski et al.'s and our "with spin" results, it is therefore necessary to add $-0.0077 \mathrm{~cm}^{-1}$ to the with spin energies in Ref. 35.

There is nearly perfect agreement between our levels and those of Ref. 35. Most differences between our energy levels and their counterparts in Ref. 35 are less than $0.0001 \mathrm{~cm}^{-1}$ which is the stated accuracy of Ref. 35. The three levels with $l_{0}=3$ have larger differences. Both with 
and without the spin term, our levels are lower by about $0.0014 \mathrm{~cm}^{-1}$. González-Martínez and Hutson[37] computed the same levels with the BOUND program[38] and also obtained levels about $0.0014 \mathrm{~cm}^{-1}$ lower than those of Ref. 35. They attribute these differences to the large $r_{0}$ tails of the corresponding wavefunctions. Our calculation supports this observation since we find it is necessary to use a larger $\max \left(r_{0}\right)=35 a_{0}$ rather than $30 a_{0}$ of Ref. 35 to converge these three levels.

Assigned values of $l_{0}$ are also given in Table I. The $l_{0}$ values are obtained from $\left\langle\boldsymbol{l}_{\mathbf{0}}^{\mathbf{2}}\right\rangle$ and are close to $l_{0}\left(l_{0}+1\right)$. Our $l_{0}$ assignments agree with those of Ref. 35; they use $L$ rather than $l_{0}$. Likewise, our $J$ and parity assignments in Table I agree with those of Ref. 35. We also give $K, m_{S}$ and $m_{N_{1}}$ assignments that are not in Ref. 35. Most of the reported bound states can be assigned to $K=0$ or 1 . However, some are a mixture of $K=0$ and 1 and the corresponding $K$ values are indicated as $0 / 1$ or $1 / 0$, with the first number being the one with a larger contribution to the wavefunction. Because the NH rotational constant is large, all the states have $N_{1}=0$. All calculated $\left\langle\boldsymbol{N}_{\mathbf{1}}^{\mathbf{2}}\right\rangle$ are smaller than $0.005 . \mathrm{NH}\left({ }^{3} \Sigma^{-}\right)$-He has a shallow potential well $\left(D_{e}=19.84 \mathrm{~cm}^{-1}\right)$ and states with $N_{1}>0$ are not bound. Due to the constraint, $K=m_{S}+m_{N_{1}}, m_{S}$ assignments are the same as $K$ assignments and therefore $m_{S}$ assignments are not given in Table I. Finally, $\left\langle\boldsymbol{j}^{\mathbf{2}}\right\rangle$ ranges from 2.002 to 2.005, indicating that all levels are $j(\mathrm{NH})=1$ states. $j(\mathrm{NH})$ is a good quantum number because spin is strongly coupled to NH rotation. As the potential well is shallow and the barrier to NH rotation is low $\left(3\right.$ to $\left.4 \mathrm{~cm}^{-1}\right)[35]$, we expect $j$ to be a good quantum number.

Finally, $\mathrm{NH}\left({ }^{3} \Sigma^{-}\right)$-He energy levels computed with method II (section VI ) and the same basis sizes agree with those computed with method I to 9 decimal places (in $\mathrm{cm}^{-1}$ ). The basis is evidently large enough that the levels are well converged despite the larger Coriolis coupling of method II. When using an iterative eigensolver, basis size is not a problem. Since the two methods deal with $S_{\zeta}^{2}$ term differently, this excellent agreement indicates that both methods are correct.

\section{RESULTS: SPIN-ROVIBRATIONAL LEVELS OF $\mathrm{O}_{2}\left({ }^{3} \Sigma_{g}^{-}\right)$-AR}

As mentioned previously, Tennyson and Mettes[10] computed $\mathrm{O}_{2}\left({ }^{3} \Sigma_{g}^{-}\right)$-Ar levels with a variational method. In this paper, we use a PES more recent than the one used by Tennyson 
and Mettes. The new PES is obtained using supermolecular unrestricted Moller-Plesset perturbation theory[39]. This computer code for this PES was kindly provided to us by Mark Severson and is deposited in the supplementary material of this paper. At equilibrium the molecule is T-shaped with $\theta_{1 e}=90^{\circ}$ and $r_{0 e}=6.7 a_{0}[39]$; the well depth is $117 \mathrm{~cm}^{-1}$. We use atomic masses; for the rotational constant of $\mathrm{O}_{2}$ in its ground vibrational state[40] we use $b_{\mathrm{O}_{2}}=1.437678 \mathrm{~cm}^{-1} ; \lambda_{0}=1.98475 \mathrm{~cm}^{-1}$ and $\gamma_{0}=-0.00845 \mathrm{~cm}^{-1}$ are, respectively, the spin-spin and spin-rotation interaction constants for $\mathrm{O}_{2}[40]$. The bend basis size is defined by $\max \left(N_{1}\right)=25$. Only odd rotational states of $\mathrm{O}_{2}$ are physically allowed and we therefore include only basis functions with odd $N_{1}$ values. 30 Gauss-Legendre quadrature points are used for $\theta_{1}$, to integrate the potential and in Eq. 19. The stretch basis for $r_{0}$ is 120 sine DVR [2] functions in the range $[3.0,35] a_{0}$. The $\mathrm{O}_{2}$ distance is fixed throughout the calculation as in the PES.[39]

To understand the pattern of the $\mathrm{O}_{2}\left({ }^{3} \Sigma_{g}^{-}\right)$-Ar levels, it is useful to discuss the $\mathrm{O}_{2}$ levels. The $N_{1}=1 \mathrm{O}_{2}$ rotational levels are split by the spin interaction term $T_{\text {fine }}$ in Eq. (4) into three levels. These three levels are from low to high, $j_{\mathrm{O}_{2}}=0,2$ and 1 levels, and are between 0 and $4 \mathrm{~cm}^{-1}$, determined by the value of $\lambda_{0}$. Here $\boldsymbol{j}_{\mathbf{O}_{2}}$ is the total angular momentum of $\mathrm{O}_{2}$. However, due to the anisotropy of the PES, $j_{\mathrm{O}_{2}}$ is not a good quantum number in $\mathrm{O}_{2}\left({ }^{3} \Sigma_{g}^{-}\right)$-Ar. Van der Avoird beautifully explained the results of Ref. 10 by using a simple model. [12] Starting from the BF basis Eq. (7) and using perturbation theory, he noted that the positive coefficient $V_{2}\left(r_{0}\right)$ of the $P_{2}(\cos \theta)$ term in the potential expansion, which is responsible for the T-shaped structure of the $\mathrm{O}_{2}$-X complex, pushes the $m_{N_{1}}=0$ levels up relative to the $m_{N_{1}}= \pm 1$ levels by $\frac{3}{5} V_{2}\left(r_{0}\right) \approx 12 \mathrm{~cm}^{-1}$. This is much larger than the magnitude of the splittings in free $\mathrm{O}_{2}$ caused by the spin interaction term $T_{\text {fine }}$. Here $m_{N_{1}}$ refers to the component of $N_{1}$ along the BF z axis. Four lower-energy "ladders" are built on six zeroth-order states obtained from products of the two $m_{N_{1}}= \pm 1$ basis functions with three spin function with $m_{S}=0, \pm 1$. The rungs of two of the ladders are doublets. The two components of each doublet have different parity. Two of the ladders have $K=0$, one has $K=1$, and one has $K=2$. These four ladders are labelled $i=1,2,3$ and 4.[12] Two higher-energy ladders are built on three zeroth-order states that are products of the $m_{N_{1}}=0$ basis function and the three spin function with $m_{S}=0, \pm 1$. One of these two ladders has $K=0$ and the other has $K=1$. The rungs of the $K=1$ ladder are doublets. These two 
ladders are labelled $i=5$ and 6.[12] No variational calculation for ladders $i=5$ and $i=6$ have been reported for $\mathrm{O}_{2}\left({ }^{3} \Sigma_{g}^{-}\right)$-Ar, but they have been reported for $\mathrm{O}_{2}\left({ }^{3} \Sigma_{g}^{-}\right)$-He.[11] The characteristics of all six ladders are summarized in Table II.

Using wavefunctions, we assign $K, m_{S}$ and $m_{N_{1}}$ values to each level. These labels make it possible to sort the levels we compute into Van der Avoird's ladders. See Table III for the ladders of assigned levels and Fig. 2 for an illustrative level diagram. Our four lower ladders including their $K, m_{S}, m_{N_{1}}$, and parity assignments agree well with those of Ref. 10, shown in Fig. 1 of Ref. 12 (most of the variational levels were not published) even though the potential energy surfaces are different. The order of the even and odd doublets for the $K>0$ ladders order also agrees. The computed $K, m_{S}, m_{N_{1}},\left\langle\boldsymbol{l}_{\mathbf{0}}^{2}\right\rangle,\left\langle\boldsymbol{N}_{\mathbf{1}}^{2}\right\rangle$ and $\left\langle\boldsymbol{j}^{2}\right\rangle$ values for each level are given in the Supplementary Material (SM). As discussed above, due to the barrier to $\mathrm{O}_{2}$ internal rotation, coupling between $\boldsymbol{N}_{\mathbf{1}}$ and $\boldsymbol{l}_{\mathbf{0}}$ is more important than coupling between $\boldsymbol{S}$ and $\boldsymbol{N}_{\mathbf{1}}$, and as a consequence neither the total rotation angular momentum $j$ of the diatomic nor the end-over-end rotation angular momentum $l_{0}$ are useful labels. $\left\langle\boldsymbol{l}_{\mathbf{0}}^{\mathbf{2}}\right\rangle$ values are not close to $l_{0}\left(l_{0}+1\right)$, as they would be if $l_{0}$ were a good quantum number, however, they do increase as one moves up the ladder. In contrast, $l_{0}$ is almost a good quantum number for $\mathrm{NH}-\mathrm{He}$ because it is closer to the free rotor limit. Because the next $\mathrm{O}_{2}$ rotational level $N_{1}=3$ is much higher in energy, all states shown are expected to have large contributions from $N_{1}=1$ basis functions. However, $\left\langle\boldsymbol{N}_{1}^{2}\right\rangle$ fluctuates between 2.7 and 4.3, far from expected value of 2 .

The ladders of $\mathrm{O}_{2}$-Ar were recently used to analyse the level structure of CO-O2. [41] In Ref. 41, CO- $\mathrm{O}_{2}$ energy levels were divided into two groups and a $K=0$ ladder (referred to as a stack in Ref. 41) in group 1 and a $K=2$ ladder in group 2 were linked with the $i=1$ ladder $(K=0)$ and the $i=2$ ladder $(K=2)$ of $\mathrm{O}_{2}$-Ar. Groups and ladders are labelled by $n\left(\mathrm{O}_{2}\right), j\left(\mathrm{O}_{2}\right)$. However, Van der Avoird[12] clearly established that $j\left(\mathrm{O}_{2}\right)$ is not a useful label for $\mathrm{O}_{2}$-Ar. It seems likely that $j\left(\mathrm{O}_{2}\right)$ is also not a useful label for $\mathrm{CO}-\mathrm{O}_{2}$.

\section{RESULTS: SPIN-ROVIBRATIONAL LEVELS OF $\mathrm{O}_{2}\left({ }^{3} \Sigma_{g}^{-}\right)$-HE}

We use an accurate PES determined with the partially spin-restricted open-shell single and double excitation coupled cluster method with perturbative triples.[42] This is a 3-D 
potential, but we fix the $\mathrm{O}_{2}$ distance at the equilibrium value, $r_{1 e}=2.282 a_{0}$. The potential well depth is $27.9 \mathrm{~cm}^{-1}$ and the equilibrium geometry is T-shaped with $\theta_{1 e}=90^{\circ}$ and $r_{0 e}=6.00 a_{0}[42]$. Compared to $\mathrm{O}_{2}$ - Ar, the $\mathrm{O}_{2}$-He potential well depth is much smaller and the rare gas atom mass is much lighter. Therefore, $\mathrm{O}_{2}$-He states are more de-localized and the Coriolis coupling has a bigger effect on energy levels, which makes it more difficult to organize the levels into ladders. The Coriolis coupling is larger for $\mathrm{O}_{2}\left({ }^{3} \Sigma_{g}^{-}\right)$-He because $B_{0}$ (see Eq. (1)) is larger. We use atomic masses and the same $\mathrm{O}_{2}$ parameters as for $\mathrm{O}_{2}\left({ }^{3} \Sigma_{g}^{-}\right)$-Ar. The bend and stretch bases are the same as for $\mathrm{O}_{2}\left({ }^{3} \Sigma_{g}^{-}\right)-\mathrm{Ar}$.

All of our calculated $\mathrm{O}_{2}\left({ }^{3} \Sigma_{g}^{-}\right)$-He bound states are given in Table IV. The ZPE is -7.47 $\mathrm{cm}^{-1}$, it sits about a quarter of the way up the well. In the same table, we also list the levels computed by Tennyson and Van der Avoird[11] (TA) using a variational method[10] and an older empirical PES[43]. The PES used by TA is certainly less accurate and its well depth is only $23.5 \mathrm{~cm}^{-1}$. On their PES, TA found 35 bound states and on the newer PES we find only 28 bound states, even though the potential we use has a deeper well. Compared to $\mathrm{O}_{2}$-Ar, the Coriolis coupling has a greater effect on the energy levels. Many of the $\mathrm{O}_{2}-\mathrm{He}$ states have more than one dominant $K$ component. Moreover, a lot of states have more than one dominant $m_{N_{1}}$. See Table IV. Because the ladders are defined by $K$ and $m_{N_{1}}$, the mixing of these quantum numbers means that it is harder to identify states with ladders.

Tennyson and Van der Avoird[11] assigned ladder labels to their calculated levels by comparing with the results of a perturbation model, but noted that the $\mathrm{O}_{2}$-He ladders were less regular than those of $\mathrm{O}_{2}$-Ar. They observed, for the first time, two higher ladders $i=5$ and $i=6$ with $m_{N_{1}}=0$ which they did not assign for $\mathrm{O}_{2}$-Ar. Using the newer PES, we are also able to identify $i=5$ and $i=6$ ladders and find that all the ladders are harder to distinguish than for $\mathrm{O}_{2}$-Ar. This is due to the stronger Coriolis coupling. The simplest way to assign a ladder label $i$ to each of our levels is to sort our level list for a fixed $J$ and fixed parity, and compare it to the assigned sorted level list of TA and use their assignments for our levels, When this is done we observe that gaps between levels in a ladder are sometimes irregular. This means that the two PESs are different enough that we cannot always use the assignments of TA and we therefore use $m_{N_{1}}, K,\left\langle\boldsymbol{j}_{\mathbf{O}_{\mathbf{2}}}^{\mathbf{2}}\right\rangle$ and $\left\langle\boldsymbol{l}_{\mathbf{0}}^{\mathbf{2}}\right\rangle$ to replace some of the TA labels attached to our levels. Once this is done the gaps between the rungs of the ladders become more regular. See Fig. 3. These re-assigned levels are marked by a star in Table IV. 
The reassignments exchange labels between ladders $i=4$ and $i=6$ and between ladders $i=1$ and $i=2$. One type of reassignment is based on values of $m_{N_{1}}$. The $i=4$ and $i=6$ ladders have $m_{N_{1}}=1$ and 0 , respectively. Thus, we reassign the the $J=1$ odd state at $-3.087 \mathrm{~cm}^{-1}$ which has $m_{N_{1}}=0$ to the $i=6$ ladder. Another type of reassignment is based on values of $K,\left\langle\boldsymbol{j}_{\mathbf{O}_{\mathbf{2}}}^{\mathbf{2}}\right\rangle$, and $\left\langle\boldsymbol{l}_{\mathbf{0}}^{\mathbf{2}}\right\rangle$. The rotation of $\mathrm{O}_{2}$ is less hindered in $\mathrm{O}_{2}$-He than in $\mathrm{O}_{2}-\mathrm{Ar}$ and therefore $\boldsymbol{j}=\boldsymbol{N}_{\mathbf{1}}+\boldsymbol{S}$, is more nearly a good quantum number. One can therefore tentatively associate ladders $i=2,3,4$ with $j_{\mathrm{O}_{2}}=2$ (5 components), ladders $i=5,6$ with $j_{\mathrm{O}_{2}}=1$ (3 components), and ladder $i=1$ with $j_{\mathrm{O}_{2}}=0$ (one component). The ladders then have the same energy order as the states of $\mathrm{O}_{2}$ for which the rotational energy order is $j_{\mathrm{O}_{2}}$ $=0,2$ and 1 .

Another example of states that are reassigned is the two $J=3$ even states at -4.703 and $-3.736 \mathrm{~cm}^{-1}$. They are re-assigned to the $i=2$ and $i=1$ ladders, respectively, for three reasons. First, their $\left\langle\boldsymbol{j}_{\mathrm{O}_{\mathbf{2}}}^{\mathbf{2}}\right\rangle$ are 5.90 and 0.38 , indicating $j_{\mathrm{O}_{2}}=2$ and 0 . Second, their $\left\langle\boldsymbol{l}_{\mathbf{0}}^{\mathbf{2}}\right\rangle$ are 2.57 and 11.73, indicating $l_{0}=1$ and 3 . If ladder $i=1$ has $j_{\mathrm{O}_{2}}=0$ then, because $\boldsymbol{J}=\boldsymbol{j}_{\mathrm{O}_{2}}+\boldsymbol{l}_{\mathbf{0}}, J=l_{0}$ and therefore a $J=3$ level must have $l_{0}=3$ and hence belong to ladder $i=1$. Third, the $K$ values of the two levels are $1 / 2 / 0$ and 0 which also supports this assignment because ladders $i=2$ and $i=1$ have $K=2$ and 1 . This example also shows that due to the rotation of $\mathrm{O}_{2}$ being less hindered, $l_{0}$ and $j_{\mathrm{O}_{2}}$ are close to being good quantum numbers for $\mathrm{O}_{2}$-He, whereas they are basically useless for $\mathrm{O}_{2}$ - $\mathrm{Ar}$, as discussed in the previous section.

One serious problem in the ladder assignment occurs for the $i=5$ ladder. The first $i=5$ state at $-3.500 \mathrm{~cm}^{-1}$ is a pure $K=0$ state as expected, but the next three $i=5$ states are all pure $K=1$ states. States with different values of $K$ should not occur in the same ladder. An illustrative level diagram for all the bound states of $\mathrm{O}_{2}\left({ }^{3} \Sigma_{g}^{-}\right)$-He is shown in Fig. 3. One can compare it with the same diagram in Ref. 11 and see that the ladder pattern is quite different. From Fig. 3, we see that level spacings between different ladders are rather different, indicating different values of end-over-end rotation constants. For all $K>0$ ladders, we find that the splitting between doublets of each rung is rather large, sometimes larger than the J-spacings within the ladder, which is in stark contrast with the $\mathrm{O}_{2}$-Ar case. 


\section{CONCLUSION}

In this paper we present a new approach for including the spin-spin term that couples rotational angular momenta and electronic spin angular momentum. It is new in two ways. First, it is new because it uses an iterative eigensolver to solve a matrix representation of the Schroedinger equation. Second, it is new because it does not expand the potential in terms of Legendre polynomials and use equations involving $3-j$ symbols to obtain matrix elements of both the potential and the spin-spin term. Instead, quadrature is used for both and matrixvector products are done by evaluating sums sequentially. The method of this paper can be used without expanding the potential and without employing angular momentum theory to derive matrix elements. This makes it easy to use with a general PES.

One might think that using quadrature when it is possible to replace matrix elements with exact closed-form equations would increase the cost of the calculation. This is not true. It is not true for the same reason that the cost of the MVPs one must evaluate when using quadrature for PES matrix elements and a SOP PES is the same. In some cases we expect the new method to be cheaper. It is certainly cheaper if, when expanded in terms of Legendre polynomials, the PES has many terms and also cheaper for molecules for which the potential matrix is large. Moreover, if the molecule of the molecule-rare gas complex were larger than a diatomic and if its shape was not fixed, then the standard approach of building and diagonalizing a matrix would be much more costly. Experiments have been

done on many $\mathrm{O}_{2}$ containing complexes, e.g. $\mathrm{N}_{2} \mathrm{O}-\mathrm{O}_{2}, \mathrm{H}_{2} \mathrm{O}-\mathrm{O}_{2}, \mathrm{HF}-\mathrm{O}_{2}$ etc. The method of this paper opens the door to doing calculations on these complexes.

\section{Supplementary Material}

See supplementary material for a list of labelled energy levels of $\mathrm{O}_{2}\left({ }^{3} \Sigma_{g}^{-}\right)$-Ar and the $\mathrm{O}_{2}\left({ }^{3} \Sigma_{g}^{-}\right)$-Ar PES code provided to us by Mark Severson.

\section{Acknowledgements}

The financial support of the Natural Sciences and Engineering Research Council is gratefully acknowledged. We thank Mark Severson for sending us the $\mathrm{O}_{2}$-Ar PES published in 
Ref. 39.

[1] T. Carrington, Jr, J. Chem. Phys. 146, 120902 (2017)

[2] J. C. Light and T. Carrington Jr. Advances in Chemical Physics 114 263-310 (2000)

[3] M.J. Bramley, J.W. Tromp, T. Carrington and G.C. Corey, J. Chem. Phys. 100, 6175 (1994)

[4] Pranab Sarkar, Nicolas Poulin, and Tucker Carrington Jr. J. Chem. Phys. 110, 10269 (1997)

[5] C. Leforestier, L. B. Braly, K. Liu, M. J. Elroy, and R. J. Saykally, J. Chem. Phys. 106, 8527 (1997)

[6] R. Chen, G. Ma, and H. Guo, Chem. Phys. Lett., 320, 567 (2000)

[7] X.-G. Wang, and T. Carrington Jr., J. Chem. Phys. 117, 6923 (2002).

[8] X.-G. Wang, T. Carrington, Jr., J. Tang, and A. R. W. McKellar, J. Chem. Phys. 123, 034301 (2005)

[9] X.-G. Wang and T. Carrington, Jr., J. Chem. Phys. 113, 044313 (2011).

[10] J. Tennyson and J. Mettes, Chem. Phys. 76, 195 (1983).

[11] J. Tennyson and A. Van Der Avoird, Chem. Phys. Lett. 105, 49 (1984).

[12] A. Van Der Avoird, J. Chem. Phys. 79, 1170 (1983).

[13] W. M. Fawzy, J. Molec. Spec. 160, 84 (1993).

[14] W. M. Fawzy, J. Molec. Spec. 191, 868 (1998).

[15] W. M. Fawzy, Comput. Phys. Comm. 181, 1789 (2010).

[16] Wafaa M. Fawzy, Christopher M. Lovejoy, David J. Nesbitt, and Jon T. Hougen J. Chem. Phys. 117, 693 (2002).

[17] Hai-Bo Qian, Dominic Seccombe, and Brian J. Howard J. Chem. Phys., 107, 7658 (1997)

[18] A. Van Der Avoird and G. Brocks, J. Chem. Phys. 87, 5346 (1987).

[19] G. Brocks, A. Van Der Avoird, B. T. Sutcliffe, and J. Tennyson, Mol. Phys. 50, 1025 (1983).

[20] R. N. Zare, Angular Momentum (Wiley: New York 1988).

[21] M. Mizushima, The theory of rotating diatomic molecules (Wiley: New York 1975).

[22] M. J. Bramley and T. Carrington, J. Chem. Phys. 99, 8519 (1993)

[23] H.-G. Yu, and J. Muckerman, J. Mol. Spectros., 214, 11 (2002)

[24] A. Csaszar, C. Fabri, T. Szidarovszky,E. Matyus, T. Furtenbacher, and G. Czako, Phys. Chem. 
Chem. Phys., 141085 (2012)

[25] J.K. Cullum and R.A. Willoughby, Lanczos algorithms for large symmetric eigenvalue computations. Volume 1, theory, Birkhauser, Boston, 1985.

[26] Matthew J. Bramley and Tucker Carrington, J. Chem. Phys. 101, 8494 (1994).

[27] X.-G. Wang and T. Carrington, Jr., J. Chem. Phys. 119, 101 (2003).

[28] J. Tennyson, Computer Physics Reports 4 1-36 (1986)

[29] M.-L. Dubernet, D. Flower, and J. M. Hutson, J. Chem. Phys. 94, 7602 (1991).

[30] J. K. G. Watson, Can. J. Phys. 46, 1637 (1968).

[31] J. T. Hougen, The Calculation of Rotational Levels and Rotational Line Intensities in Diatomic Molecules National Bureau of Standards Monograph 115, (1970).

[32] X.-G. Wang, and T. Carrington Jr., J. Chem. Phys. 146, 104105 (2015).

[33] R. N. Zare, A. L. Schrneltekopf, W. J. Harrop, and D. L. Albritton, J. Mol. Spectrosc. 46, 37 (1973).

[34] X.-G. Wang, and T. Carrington Jr., Mol. Phys. 110, 825 (2012).

[35] H. Cybulski, R. V. Krems, H. R. Sadeghpour, and A. Dalgarno J. Klos, G. C. Groenenboom, A. Van Der Avoird, D. Zgid, G. Chalasinski J. Chem. Phys. 122, 094307 (2005).

[36] C. R. Brazier, R. S. Ram and P. F. Bernath, J. Mol. Spectrosc. 120, 381 (1986).

[37] M. L. González-Martínez and J. M. Hutson Phys. Rev. A 75, 022702 (2007).

[38] J. M. Hutson, Computer code BOUND, version 5 (U.K. Engineering and Physical Sciences Research Council Collaborative Computational Project No. 6, 1993).

[39] S. M. Cybulski, R. A. Kendall, G. Chalasinski, M. W. Severson, and M. M. Szczesniak J. Chem. Phys. 106, 7731 (1997).

[40] C. Amiot and J. Verges, Can. J. Phys. 59, 1393 (1981).

[41] A. J. Barclay, A. R. W. McKellar, N. Moazzen-Ahmadi, R. Dawes, X.-G. Wangd and T. Carrington Jr. d Phys. Chem. Chem. Phys. 20, 14431 (2018).

[42] G. C. Groenenboom, and I. M. Struniewicz J. Chem. Phys. 113, 9562 (2000).

[43] M. Faubel, K. H. Kohl, J. P. Toennies, and F. A. Gianturco J. Chem. Phys. 78, 5629 (1983). 
TABLE I: Spin-rovibrational levels of $\mathrm{NH}\left({ }^{3} \Sigma^{-}\right)$-He $\left(\right.$in $\left.\mathrm{cm}^{-1}\right)$ relative to the dissociation energy with no $T_{\text {fine }}$ term. The Cybulski columns are from Ref. 35. The NH electronic ground state energy $-0.0077 \mathrm{~cm}^{-1}$ (due to $T_{\text {fine }}$ ) was added to the data of Ref. 35 to obtain the spin-rovibrational levels in the second to last column. $e / o$ is even/odd parity.

\begin{tabular}{|c|c|c|c|c|c|c|}
\hline \multirow[b]{2}{*}{$J(e / o)$} & \multirow[b]{2}{*}{$l_{0}$} & \multirow[b]{2}{*}{$K$} & \multicolumn{2}{|c|}{ no spin } & \multicolumn{2}{|c|}{ with spin } \\
\hline & & & Cybulski & This work & Cybulski & This work \\
\hline $1 e$ & 0 & $1 / 0$ & -4.4174 & -4.4175 & -4.4251 & -4.4252 \\
\hline $0 o$ & 1 & 0 & -3.7818 & -3.7819 & -3.7867 & -3.7868 \\
\hline $1 o$ & 1 & 1 & & & -3.7909 & -3.7911 \\
\hline $2 o$ & 1 & $1 / 0$ & & & -3.7892 & -3.7894 \\
\hline $1 e$ & 2 & $0 / 1$ & -2.5375 & -2.5377 & -2.5442 & -2.5444 \\
\hline $2 e$ & 2 & 1 & & & -2.5462 & -2.5464 \\
\hline $3 e$ & 2 & $1 / 0$ & & & -2.5449 & -2.5451 \\
\hline $2 o$ & 3 & $0 / 1$ & -0.7538 & -0.7552 & -0.7613 & -0.7627 \\
\hline $3 o$ & 3 & 1 & & & -0.7619 & -0.7633 \\
\hline $4 o$ & 3 & $1 / 0$ & & & -0.7614 & -0.7628 \\
\hline
\end{tabular}


TABLE II: Labels of ladders[12] for spin-rovibrational levels of $\mathrm{O}_{2}-\operatorname{Ar}\left({ }^{3} \Sigma^{-}\right) . \quad \sigma=(e, f)$ is the spectroscopic parity with $e$ and $f$ representing $(-1)^{J+P}=+1$ and -1 , respectively.

\begin{tabular}{cccccc}
\hline \hline ladder & $K(\sigma)$ & $m_{S}$ & $m_{N_{1}}$ & $N_{1}$ & $(-1)^{J+S+P}$ \\
\hline$i=1$ & $0(f)$ & 1 & 1 & 1 & +1 \\
$i=2$ & $2(e, f)$ & 1 & 1 & 1 & \pm 1 \\
$i=3$ & $1(e, f)$ & 0 & 1 & 1 & \pm 1 \\
$i=4$ & $0(e)$ & 1 & 1 & 1 & -1 \\
$i=5$ & $0(f)$ & 0 & 0 & 1 & +1 \\
$i=6$ & $1(e, f)$ & 1 & 0 & 1 & \pm 1 \\
\hline \hline
\end{tabular}


TABLE III: Spin-rovibrational levels of $\mathrm{O}_{2}-\operatorname{Ar}\left({ }^{3} \Sigma_{g}^{-}\right)\left(\right.$in $\left.\mathrm{cm}^{-1}\right)$ relative to the dissociation energy without $T_{\text {fine }}$. We follow Ref. 12 and label the ladders by $i$ in the first row. See also Table II and Fig. 2 for more information about the ladders.

\begin{tabular}{|c|c|c|c|c|c|c|c|c|c|}
\hline \multirow[b]{2}{*}{$J$} & \multirow{2}{*}{$\begin{array}{l}i=1 \\
\mathrm{~K}=0 \mathrm{f}\end{array}$} & \multicolumn{2}{|c|}{$i=2$} & \multicolumn{2}{|c|}{$i=3$} & \multirow{2}{*}{$\begin{array}{c}i=4 \\
\mathrm{~K}=0 \mathrm{e}\end{array}$} & \multirow{2}{*}{$\begin{array}{l}i=5 \\
\mathrm{~K}=0 \mathrm{f}\end{array}$} & \multicolumn{2}{|c|}{$i=6$} \\
\hline & & $\mathrm{K}=2 \mathrm{e}$ & $K=2 f$ & $\mathrm{~K}=1 \mathrm{e}$ & $\mathrm{K}=1 \mathrm{f}$ & & & $\mathrm{K}=1 \mathrm{e}$ & $\mathrm{K}=1 \mathrm{f}$ \\
\hline 0 & -88.934 & & & & & -85.182 & -79.484 & & \\
\hline 1 & -88.802 & & & -85.454 & -85.411 & -85.001 & -79.380 & -77.521 & -77.507 \\
\hline 2 & -88.538 & -87.212 & -87.212 & -85.231 & -85.117 & -84.656 & -79.170 & -77.28 & -77.239 \\
\hline 3 & -88.143 & -86.830 & -86.828 & -84.878 & -84.677 & -84.156 & -78.853 & -76.919 & -76.839 \\
\hline 4 & -87.617 & -86.321 & -86.313 & -84.389 & -84.093 & -83.510 & -78.428 & -76.438 & -76.309 \\
\hline 5 & -86.962 & -85.682 & -85.666 & -83.762 & -83.366 & -82.720 & -77.894 & -75.837 & -75.651 \\
\hline 6 & -86.178 & -84.915 & -84.885 & -82.998 & -82.499 & -81.787 & -77.248 & -75.116 & -74.866 \\
\hline 7 & -85.268 & -84.018 & -83.966 & -82.095 & -81.492 & -80.713 & -76.489 & -74.277 & -73.958 \\
\hline 8 & -84.231 & -82.991 & -82.910 & -81.055 & -80.348 & -79.499 & -75.616 & -73.318 & -72.926 \\
\hline 9 & -83.070 & -81.833 & -81.713 & -79.878 & -79.069 & -78.147 & -74.628 & -72.242 & -71.774 \\
\hline 10 & -81.786 & -80.545 & -80.377 & -78.565 & -77.656 & -76.656 & -73.524 & -71.047 & -70.503 \\
\hline
\end{tabular}


TABLE IV: All bound spin-rovibrational levels of $\mathrm{O}_{2}-\mathrm{He}\left({ }^{3} \Sigma_{g}^{-}\right)\left(\mathrm{in}^{-1}\right)$ relative to the dissociation energy without $T_{\text {fine }}$. TA refers to Tennyson and van der Avoird[11]. TW is This Work. To the TA levels of Ref. 11, we have added $0.246 \mathrm{~cm}^{-1}$, the energy of the $J=1 \mathrm{O}_{2}$ state, to account for the difference in the definition of the zero of energy in this paper and in TA. All levels in this table are below $0.246 \mathrm{~cm}^{-1}$ and are bound, except for one level at $0.248 \mathrm{~cm}^{-1}$.

\begin{tabular}{|c|c|c|c|c|c|c|c|c|c|}
\hline$E$ & $E(\mathrm{TA})$ & $K$ & $m_{S}$ & $m_{N_{1}}$ & $\left\langle l_{0}^{2}\right\rangle$ & $\left\langle N_{1}^{2}\right\rangle$ & $\left\langle\boldsymbol{j}_{\mathrm{O}_{2}}^{2}\right\rangle$ & $\begin{array}{c}\text { ladder } \\
(\mathrm{TA})\end{array}$ & $\begin{array}{l}\text { ladder } \\
(\mathrm{TW})\end{array}$ \\
\hline \multicolumn{10}{|l|}{$\mathrm{J}=0$, even } \\
\hline-3.142 & -5.515 & 0 & 1 & 1 & 2.16 & 2.16 & 2.16 & $i=4$ & $i=4$ \\
\hline \multicolumn{10}{|l|}{$\mathrm{J}=0$, odd } \\
\hline-7.471 & -9.598 & 0 & 1 & 1 & 0.25 & 2.15 & 0.25 & $i=1$ & $i=1$ \\
\hline-3.500 & -4.484 & 0 & 0 & 0 & 5.81 & 2.39 & 5.81 & $i=5$ & $i=5$ \\
\hline \multicolumn{10}{|l|}{$\mathrm{J}=1$, even } \\
\hline-6.855 & -9.027 & 0 & 1 & 1 & 2.19 & 2.15 & 0.29 & $i=1$ & $i=1$ \\
\hline-4.534 & -6.287 & $1 / 0$ & 0 & $0 / 1$ & 2.10 & 2.26 & 5.83 & $i=3$ & $i=3$ \\
\hline-2.719 & -4.262 & 1 & $1 / 0$ & $1 / 0$ & 2.46 & 2.13 & 2.36 & $i=5$ & $i=5$ \\
\hline-1.650 & -2.897 & $0 / 1$ & $1 / 0$ & 0 & 11.36 & 2.29 & 5.75 & $i=6$ & $i=6$ \\
\hline \multicolumn{10}{|l|}{$\mathrm{J}=1$, odd } \\
\hline-3.670 & -6.032 & 1 & 0 & 1 & 1.94 & 2.16 & 3.34 & $i=3$ & $i=3$ \\
\hline-3.087 & -4.950 & 1 & 1 & 0 & 4.39 & 2.15 & 4.80 & $i=4$ & $i=6^{*}$ \\
\hline-1.708 & -3.435 & $0 / 1$ & 1 & 1 & 5.99 & 2.12 & 2.20 & $i=6$ & $i=4^{*}$ \\
\hline \multicolumn{10}{|c|}{$\mathrm{J}=2$, even } \\
\hline-4.971 & -7.303 & 2 & 1 & 1 & 2.20 & 2.36 & 6.13 & $i=2$ & $i=2$ \\
\hline-2.904 & -5.134 & $1 / 0$ & $1 / 0$ & 1 & 2.42 & 2.14 & 2.26 & $i=3$ & $i=3$ \\
\hline-1.541 & -3.520 & 1 & $1 / 0$ & $1 / 0$ & 11.72 & 2.20 & 5.94 & $i=4$ & $i=6^{*}$ \\
\hline 0.122 & -1.805 & $0 / 1$ & 1 & 1 & 11.96 & 2.11 & 2.16 & $i=6$ & $i=4^{*}$ \\
\hline \multicolumn{10}{|c|}{$\mathrm{J}=2$, odd } \\
\hline-5.701 & -8.013 & 0 & 1 & 1 & 4.64 & 2.21 & 1.70 & $i=1$ & $i=1$ \\
\hline-5.295 & -7.342 & $2 / 0 / 1$ & 1 & 1 & 1.83 & 2.26 & 4.65 & $i=2$ & $i=2$ \\
\hline-3.584 & -5.357 & $2 / 0 / 1$ & $1 / 0$ & 1 & 5.95 & 2.33 & 5.96 & $i=3$ & $i=3$ \\
\hline-1.514 & -3.198 & 1 & $1 / 0$ & $0 / 1$ & 6.09 & 2.11 & 2.20 & $i=5$ & $i=5$ \\
\hline \multicolumn{10}{|l|}{$\mathrm{J}=3$, even } \\
\hline-4.703 & -7.303 & $1 / 2 / 0$ & 1 & 1 & 2.57 & 2.32 & 5.90 & $i=1$ & $i=2^{*}$ \\
\hline-3.736 & -5.134 & 0 & 1 & 1 & 11.73 & 2.10 & 0.38 & $i=2$ & $i=1^{*}$ \\
\hline-1.817 & -3.520 & $2 / 0$ & 1 & 1 & 11.98 & 2.35 & 6.04 & $i=3$ & $i=3$ \\
\hline 0.248 & -1.805 & 1 & $1 / 0$ & $0 / 1$ & 11.93 & 2.10 & 2.19 & $i=5$ & $i=5$ \\
\hline \multicolumn{10}{|l|}{$\mathrm{J}=3$, odd } \\
\hline-3.635 & -5.987 & 2 & 1 & 1 & 6.17 & 2.33 & 6.12 & $i=2$ & $i=2$ \\
\hline-1.640 & -3.814 & $1 / 0$ & 1 & 1 & 6.20 & 2.13 & 2.17 & $i=3$ & $i=3$ \\
\hline \multicolumn{10}{|c|}{$\mathrm{J}=4$, even } \\
\hline-1.737 & -4.128 & 2 & 1 & 1 & 12.12 & 2.30 & 6.11 & $i=2$ & $i=2$ \\
\hline 0.192 & -1.990 & $1 / 0$ & 1 & 1 & 12.12 & 2.11 & 2.14 & $i=3$ & $i=3$ \\
\hline \multicolumn{10}{|l|}{$\mathrm{J}=4$, odd } \\
\hline-3.414 & -5.484 & $1 / 0 / 2$ & 1 & 1 & 6.23 & 2.30 & 6.08 & $i=1$ & $i=2^{*}$ \\
\hline-1.415 & -3.534 & 0 & 1 & 126 & 19.93 & 2.09 & 0.16 & $i=2$ & $i=1^{*}$ \\
\hline \multicolumn{10}{|c|}{$\mathrm{J}=5$, even } \\
\hline-1.598 & -3.628 & $1 / 0$ & 1 & 1 & 12.13 & 2.29 & 6.09 & $i=1$ & $i=2^{*}$ \\
\hline
\end{tabular}




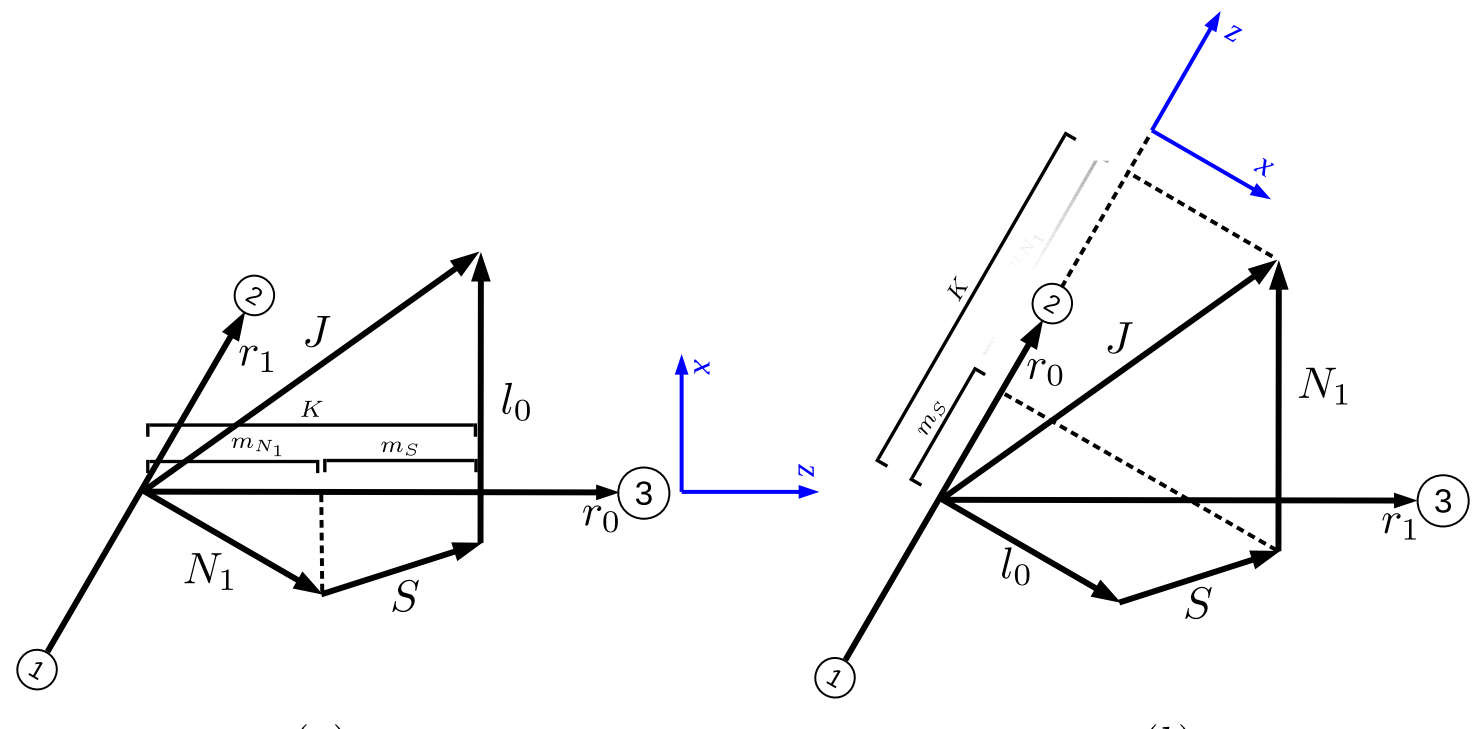

(a)

(b)

FIG. 1: Two coupling schemes used for systems consisting of an open shell $\Sigma$ diatomic molecule and a closed shell atom, such as $\mathrm{O}_{2}$-Ar or NH-Ar. In scheme (a), the vector $r_{0}$ is associated with the intermonomer Jacobi vector. In scheme (b), the vector $r_{0}$ is associated with the diatomic vector. The $z$ axes of the body-fixed (BF) (marked in blue) and dimer-fixed frames in both schemes are along $\boldsymbol{r}_{\mathbf{0}}$ and the $x$-axes of the BF frames are along $\boldsymbol{r}_{\mathbf{0}} \times \boldsymbol{r}_{\mathbf{1}} \times \boldsymbol{r}_{\mathbf{0}}$. 


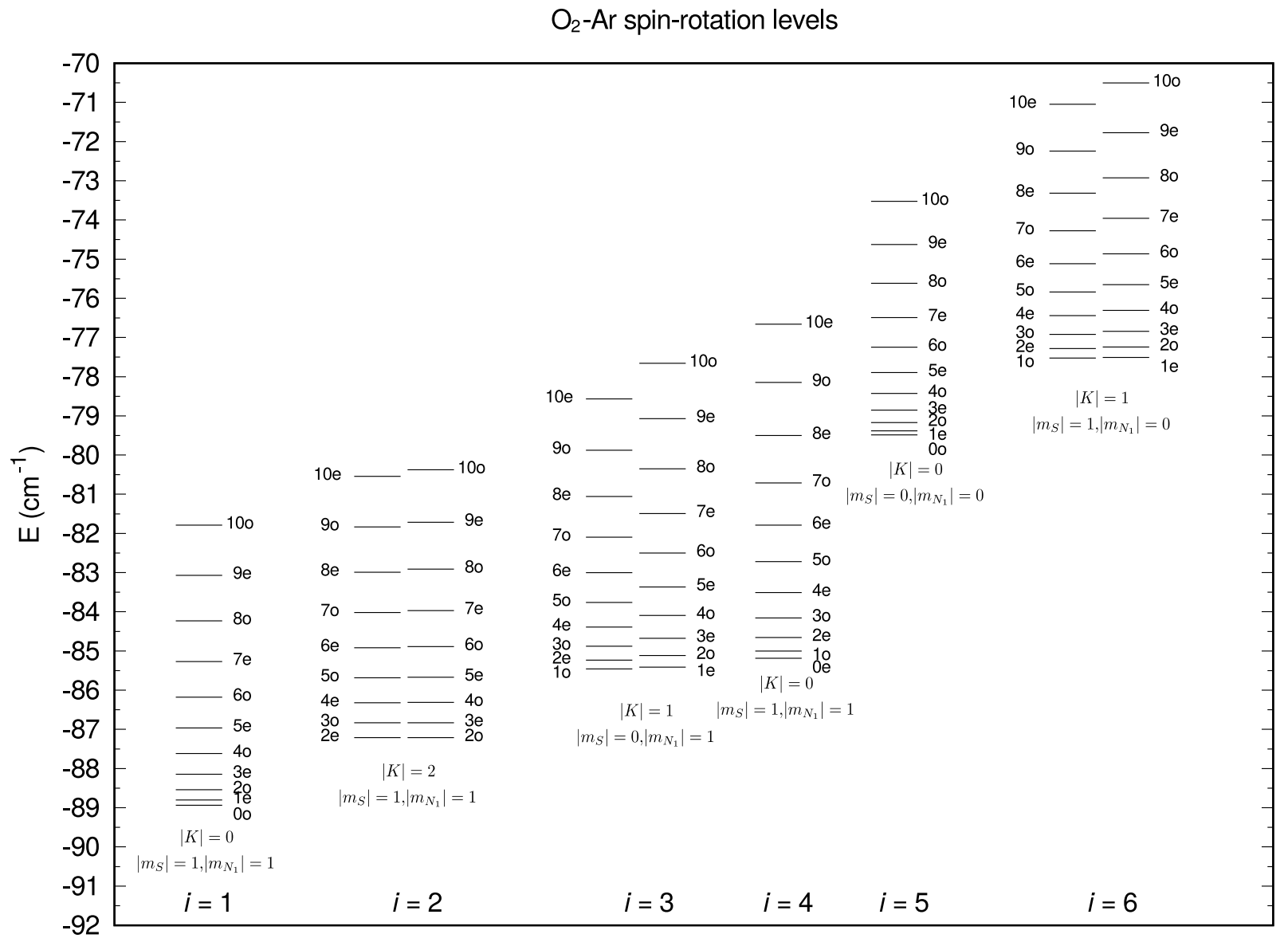

FIG. 2: Fine-structure rovibrational levels of $\mathrm{O}_{2}$-Ar. Theoretical levels are sorted into ladders based on the approximate quantum numbers $K, m_{S}$, and $m_{N_{1}}$. The first four ladders $i=1, \cdots, 4$ originate from $m_{N_{1}}=1$ states of $\mathrm{O}_{2}$. The next two ladders $i=5,6$ originate from $m_{N_{1}}=0$ states of $\mathrm{O}_{2}$. Indicated with each level is $J(e / o)$ where $e / o$ is even/odd parity. 


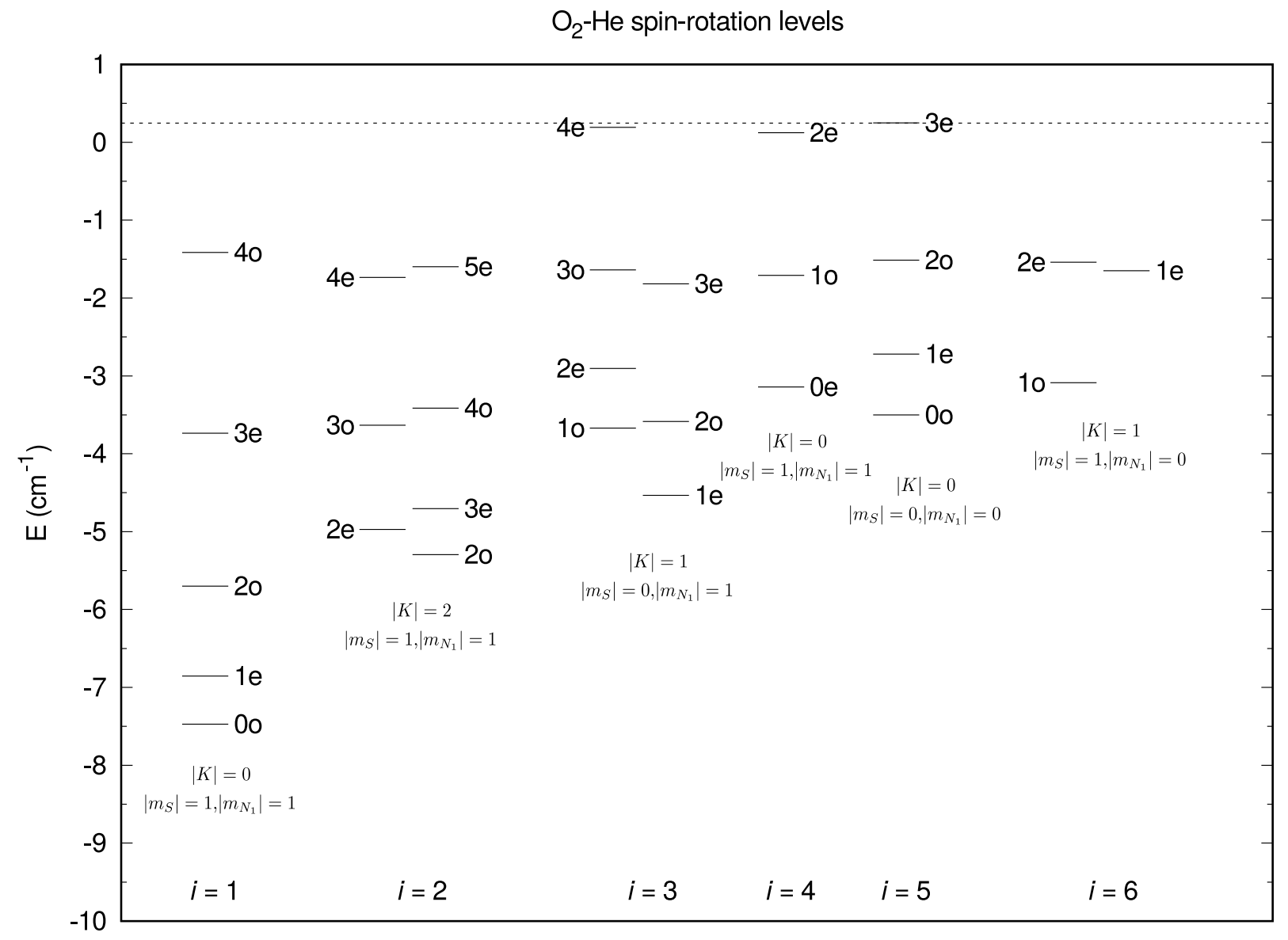

FIG. 3: Fine-structure rovibrational levels of $\mathrm{O}_{2}$-He. See the caption of Fig. 2 for additional information about the ladders. The dashed line is the dissociation limit with $\mathrm{O}_{2}$ in its $J=1$ rotational state with energy $0.246 \mathrm{~cm}^{-1}$. 